\title{
ESTIMATIVA DA ÁREA FOLIAR DE LIMOEIRO-CRAVO USANDO DIMENSÕES LINEARES DO LIMBO FOLIAR
}

SILVA, Raimundo Thiago Lima da ${ }^{1}$ ANDRADE, Ariele Carneiro de ${ }^{2}$ OLIVEIRA, Lillian Matias de ${ }^{2}$

LIMA, Layla Gerusa Souza ${ }^{2}$ OLIVEIRA, Raimundo Leonardo Lima de ${ }^{2}$ MELO, Émile Costa ${ }^{3}$ OLIVEIRA NETO, Cândido Ferreira de ${ }^{4}$

\begin{abstract}
RESUMO: Com o objetivo de obter equações que, por meio de parâmetros lineares dimensionais das folhas, permita a estimativa da área foliar de Limoeiro-Cravo (Citrus limonia (L.) Osbeck), foi conduzido um experimento em uma propriedade rural no município de Capitão Poço - PA. A área foliar foi determinada pelo método dos discos. Ajustaram-se modelos lineares, lineares sem intercepto, quadráticos, cúbicos, logarítmicos, exponenciais e potenciais entre área foliar e o comprimento, a largura, a soma (comprimento + largura) e seus produtos (comprimento x largura), sendo eliminados os que apresentaram coeficiente de determinação menor do que 0,80. A estatística utilizada para avaliar o desempenho dos modelos foi o coeficiente de correlação de Pearson (r), o de determinação $\left(\mathrm{R}^{2}\right)$, a raiz do quadrado médio do erro, o erro absoluto médio, o índice d de Willmott e o índice CS. Os modelos que melhor se ajustaram aos dados foram: o linear, o linear sem intercepto, o potencial e o quadrático, considerando a relação (comprimento x largura) como variável independente.
\end{abstract}

Palavras-chave: Coeficiente de determinação. Citrus limonia (L.) Osbeck.. Dimensões do limbo foliar.

\section{LEAF AREA ESTIMATE OF RANGPUR LIME USING LINEAR DIMENSIONS OF THE LEAF BLADE}

SUMMARY: In order to obtain equations which, through dimensional linear parameters of the leaves, allows the estimation of leaf area of rangpur lime (Citrus limonia (L.) Osbeck) an experiment was conducted in a rural area of Capitão Poço - PA. Leaf area was determined by the method of discs. Linear, linear without intercept, quadratic, cubic, logarithmic, exponential and potential models were adjusted between leaf area and length, the width, the sum (length + width) and its products (length $\mathrm{x}$ width), and eliminated those that had a coefficient of determination less than 0.80. The statistic used to evaluate the performance of the models was the Pearson correlation coefficient $(\mathrm{r})$, the determination $\left(\mathrm{R}^{2}\right)$, the root mean square error, mean absolute error, the index $\mathrm{d}$ of Willmott and CS index. The models that best fit were: the linear, linear without intercept, the potential and the quadratic, considering the ratio (length $\mathrm{x}$ width) as the independent variable.

Keywords: Coefficient of determination. Citrus limonia (L.) Osbeck. Dimensions of the leaf.

\section{INTRODUÇÃO}

O limoeiro 'Cravo' (Citrus limonia (L.) Osbeck), é uma árvore frutífera de folhas perenes da família das rutáceas, a mesma da laranjeira, da tangerineira e da limeira. O limão provavelmente é uma das frutas mais conhecidas e usadas do mundo, e através dele preparam-se refrigerantes, sorvetes, molhos, temperos, aperitivos, remédios, xaropes, produtos de limpeza e outros (TONET et al., 2010).

\footnotetext{
${ }^{1}$ Eng. Agrônomo formado pela Universidade Federal Rural da Amazônia, Mestre em Engenharia Agrícola pela Universidade Federal de Santa Maria e Doutorando em Agronomia - Fitotecnia pela Universidade Federal do Ceará.

${ }^{2}$ Graduanda em Agronomia - Campus Capitão Poço

${ }^{3}$ Eng. Agrônomo - UFRA

${ }^{4}$ Professor Dr. Adjunto de Fisiologia Vegetal - UFRA - Campus De Capitão Poço
} 
As principais funções das folhas nas plantas são a interceptação e absorção da luz solar e a sua capacidade fotossintética (LARCHER, 2000; TAIZ ; ZEIGER, 2004; SEVERINO et. al., 2004). A folha é o principal órgão no processo respiratório e responsável pelas trocas gasosas entre a planta e o ambiente (PEREIRA et. al., 1997). O conhecimento da superfície foliar é de grande utilidade para a avaliação de técnicas culturais como poda, adubação, densidade de plantio, evapotranspiração, irrigação, interceptação de luz, eficiência fotossintética e aplicação de defensivos (BLANCO ; FOLLEGATTI, 2005; LUCENA et al., 2011).

Existem inúmeras possibilidades de se determinar a área foliar de uma planta, através de métodos destrutivos e não destrutivos, porém muitos são inadequados por serem destrutivos e/ou por dependerem de aparelhos disponíveis somente em laboratórios ou, ainda, por demandarem excessiva mão-de-obra para execução. A área foliar é uma das características mais difíceis de serem mensuradas (BIANCO et al., 2007)

A área foliar pode ser estimada utilizando parâmetros dimensionais de folhas, os quais apresentam boas correlações com a superfície foliar por meio de equações de regressão entre a área foliar real e os parâmetros dimensionais lineares das folhas. Pela facilidade e por ser não-destrutivo, os modelos mais utilizados são os que usam comprimento da nervura principal, a largura máxima e as relações entre essas medidas (LIMA et al., 2008). Esse método já foi utilizado, com sucesso, para inúmeras plantas, tais como Crambe abyssinica (TOEBE et al., 2010), Cissampelos glaberrima (BIANCO et al., 2002), Typha latifolia (BIANCO et al., 2003), Tridax procumbens (BIANCO et al., 2004), Sesamum indicum L. (SILVA et al., 2002), Beta vulgaris L. (MARROCOS et al., 2010), Phaseolus vulgaris L. (QUEIROGA et al., 2003) entre outras.

O presente trabalho teve como objetivo determinar modelos matemáticos eficientes, para estimar a área foliar de limoeiro ‘Cravo', utilizando-se de parâmetros dimensionais de folhas de diferentes tamanhos.

\section{MATERIAL E MÉTODOS}

O presente trabalho foi desenvolvido em um pomar com 1200 plantas de limoeiro 'Cravo', na idade de cinco anos, da cultivar cravo, distribuídas no espaçamento 5 X $5 \mathrm{~m}$, localizado no município de Capitão Poço na microrregião do Guamá no Estado do Pará, situado na latitude: $01^{\circ} 41^{\prime} \mathrm{S}$, longitude: $047^{\circ} 06^{\prime}$ W e altitude média da área em torno de $73 \mathrm{~m}$. O clima da região, segundo a classificação de Köppen, é do tipo Am com precipitação anual em torno de $2.500 \mathrm{~mm}$, com uma curta estação seca entre setembro e novembro (precipitação mensal em torno de $60 \mathrm{~mm}$ ), temperatura média de $26^{\circ}$ e umidade relativa do ar entre $75 \%$ e $89 \%$ nos meses com menor e maior precipitação, respectivamente (SCHWART, 2007).

A adubação orgânica anual utilizada foi de $5 \mathrm{Kg}$ de esterco bovino por planta, já a adubação química foi realizada com o fertilizante NPK (10-28-20), na quantidade de 500 g por planta. A fim de controlar a proliferação de plantas daninhas na área durante o cultivo, fez-se o uso do herbicida glifosato e de capinas manuais.

$\mathrm{Na}$ determinação da área foliar (AF), o primeiro passo foi à amostragem aleatória de 368 folhas, coletadas na fase de florescimento da cultura, de modo que fosse possível coletar folhas pequenas ( $\mathrm{AF} \leq$ $\left.25 \mathrm{~cm}^{2}\right)$, médias $\left(25<\mathrm{AF} \leq 50 \mathrm{~cm}^{2}\right)$ e grandes $\left(\mathrm{AF}>50 \mathrm{~cm}^{2}\right)$. É importante salientar que todas as folhas não tinham qualquer dano ou ataque de doenças ou pragas e que se encontrava em franco desenvolvimento vegetativo.

No laboratório multifuncional da Universidade Federal Rural da Amazônia, Campus de Capitão Poço, as 368 folhas, foram medidas, coletando-se então o comprimento ao longo da nervura central (C) e a largura máxima perpendicular a nervura central (L) do limbo foliar, com régua milimetrada. E então foi calculado o produto do comprimento pela largura $(\mathrm{CxL})$ do limbo foliar. Em seguida com auxílio de um calador com diâmetro de $25 \mathrm{~mm}$, obteve-se o número máximo possível de discos do limbo, incluindo as nervuras. Cada disco tinha uma área de $4,9 \mathrm{~cm}^{2}$. 
Individualmente cada amostra de discos e o restante da folha foram acondicionados separadamente em sacos de papel e levados à estufa de secagem, a uma temperatura de $65^{\circ} \mathrm{C}$, até peso constante.

Para a determinação da massa de matéria seca das duas partes foi utilizada uma balança eletrônica de precisão com quatro casas decimais. A área total de cada folha (AF), $\mathrm{em} \mathrm{cm}^{2}$, foi calculada através da (Eq. 1):

$$
A F=\frac{(M S f * A T d)}{M S d}
$$

Em que MSd - é a massa seca dos discos, ATd - área total dos discos e MSf - a massa seca total das folhas.

O passo seguinte foi geração de uma sequência de números aleatórios para a seleção de 188 folhas, que foram separadas e utilizadas na validação dos modelos. Com os dados de C, L, C x L e da AF das 180 folhas restantes, modelou-se a área foliar, determinada pelo método dos discos (Y) em função do $\mathrm{C}$, da $\mathrm{L}$ e do $\mathrm{C} \times \mathrm{L}$, por meio dos modelos: quadrático $\left(Y=a+b x+c x^{2}\right)$, cúbico $\left(Y=a+b x+c x^{2}+d x^{3}\right)$, potência $\left(Y=a x^{b}\right)$, linear $(\mathrm{Y}=\mathrm{a}+\mathrm{bx})$ e linear sem intercepto $(\mathrm{Y}=\mathrm{bx})$.

Para a validação dos modelos de estimativa de área foliar, foram utilizados 188 valores estimados por cada modelo (Ye) e 188 valores observados (Yo). Para cada modelo, foi ajustada uma regressão linear simples $(\mathrm{Ye}=\mathrm{a}+\mathrm{bYo})$ da área foliar estimada pelo modelo (variável dependente) em função da área foliar observada (variável independente).

E por fim foram calculados os coeficientes de correlação linear de Pearson (r) por meio do teste $\mathrm{t}$ de Student a $1 \%$ de probabilidade de erro e de determinação $\left(R^{2}\right)$ entre Ye e Yo. Também foi calculado o erro absoluto médio (EAM), a raiz do quadrado médio do erro (RQME), o índice d de Willmott (WILLMOTT, 1981) e o índice CS (CAMARGO ; SENTELHAS, 1997), por meio, respectivamente, das (Eqs. 2, 3, 4 e 5 ).

$$
\begin{gathered}
E A M=\frac{\sum_{i=1}^{n}|Y e-Y o|}{n} \\
R Q M E=\sqrt{\frac{\sum_{i=1}^{n}(Y e-Y o)^{2}}{n}} \\
d=1-\left(\begin{array}{c}
\sum_{i=1}^{n} \boldsymbol{Q} e-Y o_{-}^{叉} \\
\sum_{i=1}^{n}\left(Y e-\bar{Y}|+| Y o-\left.\bar{Y}\right|^{2}\right.
\end{array}\right) \\
C S=r . d
\end{gathered}
$$

Sendo Ye - os valores estimados de área foliar, Yo - os valores observados de área foliar por meio do método dos discos, $\bar{Y}$ - a média dos valores observados e n - é o número de folhas (n=188).

Os melhores modelos de estimativa de área foliar de limoeiro-cravo em função do $\mathrm{C}$, da $\mathrm{L}$ e da $\mathrm{C} \times \mathrm{L}$ do limbo foliar, segue os seguintes critérios: coeficientes de correlação linear de Pearson (r) e de determinação $\left(\mathrm{R}^{2}\right)$ mais próximos de um, erro absoluto médio (EAM) e raiz do quadrado médio do erro (RQME) mais próximo de zero, e índices d de Willmott e CS mais próximos de um. As análises estatísticas dos resultados do experimento foram realizadas utilizando o aplicativo Office Excel e o programa ASSISTAT versão 7.6 beta.

\section{RESULTADOS E DISCUSSÃO}

Os valores médios para comprimento do limbo, largura do limbo e área foliar real, foram de 9,416 cm, $5,455 \mathrm{~cm}$, e 43,332 cm², respectivamente, nas 180 folhas utilizadas na construção dos modelos de estimativa de 
área foliar. De todos os modelos gerados, apenas 13 apresentaram coeficientes de determinação elevados $\left(\mathrm{R}^{2}>\right.$ 0,80 ), indicando que pelo menos $80 \%$ das variações observadas na área foliar foram explicadas pelas equações obtidas, como mostra a tabela 1 .

Tabela 1. Modelos de regressão para estimativa da área foliar $\left(\mathrm{AF}, \mathrm{cm}^{2}\right)$ de limoeiro e respectivos coeficientes de determinação de geração do modelo $\left(\mathrm{R}^{2}\right)$, em função da soma $(\mathrm{C}+\mathrm{L})$ e do produto $(\mathrm{CxL})$ do comprimento (C) e largura máxima (L) do limbo foliar.

\begin{tabular}{clc}
\hline $\mathrm{N}^{\mathrm{o}}$ & \multicolumn{1}{c}{ Modelos } & $\mathrm{R}^{2}$ \\
\hline 1 & $\mathrm{AF}=0.219(\mathrm{C}+\mathrm{L})^{2}-1.1689(\mathrm{C}+\mathrm{L})+8.0926$ & 0.856 \\
2 & $\mathrm{AF}=81.578 \mathrm{Ln}(\mathrm{C}+\mathrm{L})-179.65$ & 0.826 \\
3 & $\mathrm{AF}=5.5175(\mathrm{C}+\mathrm{L})-42.187$ & 0.848 \\
4 & $\mathrm{AF}=0.0227(\mathrm{C}+\mathrm{L})^{3}-0.7935(\mathrm{C}+\mathrm{L})^{2}+13.63(\mathrm{C}+\mathrm{L})-62.797$ & 0.857 \\
5 & $\mathrm{AF}=0.1688(\mathrm{C}+\mathrm{L})^{2.0174}$ & 0.882 \\
6 & $\mathrm{AF}=5.2297 \mathrm{e}^{0.1342(\mathrm{C}+\mathrm{L})}$ & 0.876 \\
7 & $\mathrm{AF}=0.0022(\mathrm{CxL})^{2}+0.5461(\mathrm{CxL})+4.4888$ & 0.893 \\
8 & $\mathrm{AF}=42.204 \mathrm{Ln}(\mathrm{CxL})-126.29$ & 0.854 \\
9 & $\mathrm{AF}=0.8003(\mathrm{CxL})-2.4636$ & 0.891 \\
10 & $\mathrm{AF}=0.00009(\mathrm{CxL})^{3}-0.0136(\mathrm{CxL})^{2}+1.3964(\mathrm{CxL})-9.9588$ & 0.895 \\
11 & $\mathrm{AF}=0.7593(\mathrm{CxL})$ & 0.889 \\
12 & $\mathrm{AF}=0.6358(\mathrm{CxL})^{1.042}$ & 0.908 \\
13 & $\mathrm{AF}=14.01 \mathrm{e}^{0.0191(\mathrm{CxL})}$ & 0.889 \\
\hline
\end{tabular}

Quando se considerou apenas uma dimensão comprimento ou largura máxima do limbo foliar, os modelos matemáticos estimadores de área foliar não apresentaram ajuste satisfatório, com coeficiente de determinação $\left(\mathrm{R}^{2}<0,8\right)$. Resultados similares foram obtidos por Monteiro et al. (2005), os quais ao realizarem análises de regressão da área foliar de algodoeiro com o comprimento e a largura das folhas separadamente, encontraram menores ajustes do que aqueles realizados com o produto das duas dimensões.

Os modelos linear, linear sem intercepto, quadrático, cúbico, potencial e exponencial que utilizam a relação $(\mathrm{CxL})$ do limbo foliar, apresentaram valores de coeficientes de determinação $\left(\mathrm{R}^{2}\right)$ superiores aos demais. O melhor resultado foi obtido com o modelo potencial, com $\mathrm{R}^{2}=0,908$.

Os modelos mais precisos foram o linear, o linear sem intercepto, o quadrático e o potencial, que utilizaram a $(\mathrm{C} \times \mathrm{L})$ na estimativa da área foliar, com coeficientes de correlação linear de Pearson (r) e de determinação $\left(\mathrm{R}^{2}\right)$ mais próximos de um, menor erro absoluto médio (EAM) e raiz do quadrado médio do erro (RQME) e, índices de de Willmott e CS mais próximos de um, (Tabela 2).

Tabela 2. Variáveis independentes (x), coeficientes de correlação linear de Pearson $(r)$ e de determinação $\left(\mathrm{R}^{2}\right)$, obtidos na regressão linear ajustada entre a área foliar estimada (variável dependente) e a observada (variável independente). Erro absoluto médio (EAM), raiz do quadrado médio do erro (RQME), índice d de Willmott e índice CS, calculados com base nas áreas foliares, observada e estimada de 188 folhas de limoeiro.

(Continua)

\begin{tabular}{llccccccc}
\hline $\mathrm{N}^{\mathrm{o}}$ & \multicolumn{1}{c}{ Modelo } & $\mathrm{x}$ & $\mathrm{r}$ & $\mathrm{R}^{2}$ & $\mathrm{EAM}$ & $\mathrm{RQME}$ & $\mathrm{d}$ & $\mathrm{CS}$ \\
\hline 1 & Linear & $\mathrm{CxL}$ & $0.916^{*}$ & 0.840 & 3.873 & 5.441 & 0.954 & 0.874 \\
2 & Linear sem intercepto & $\mathrm{CxL}$ & $0.916^{*}$ & 0.840 & 3.848 & 5.434 & 0.952 & 0.872 \\
3 & Logarítmico & $\mathrm{CxL}$ & $0.903^{*}$ & 0.816 & 4.356 & 6.037 & 0.934 & 0.844 \\
4 & Quadrático & $\mathrm{CxL}$ & $0.914^{*}$ & 0.836 & 3.939 & 5.559 & 0.954 & 0.872 \\
5 & Cúbico & $\mathrm{CxL}$ & $0.906^{*}$ & 0.820 & 4.192 & 5.954 & 0.948 & 0.858 \\
6 & Potencial & $\mathrm{CxL}$ & $0.916^{*}$ & 0.839 & 3.906 & 5.478 & 0.953 & 0.873 \\
7 & Exponencial & $\mathrm{CxL}$ & $0.895^{*}$ & 0.801 & 4.849 & 7.105 & 0.937 & 0.839
\end{tabular}


Tabela 2. Variáveis independentes (x), coeficientes de correlação linear de Pearson (r) e de determinação $\left(\mathrm{R}^{2}\right)$, obtidos na regressão linear ajustada entre a área foliar estimada (variável dependente) e a observada (variável independente). Erro absoluto médio (EAM), raiz do quadrado médio do erro (RQME), índice d de Willmott e índice CS, calculados com base nas áreas foliares, observada e estimada de 188 folhas de limoeiro.

(Conclusão)

\begin{tabular}{cllllllll}
8 & Linear & $\mathrm{C}+\mathrm{L}$ & $0.904^{*}$ & 0.818 & 4.401 & 5.989 & 0.938 & 0.848 \\
9 & Logarítmico & $\mathrm{C}+\mathrm{L}$ & $0.894^{*}$ & 0.799 & 4.752 & 6.440 & 0.923 & 0.825 \\
10 & Quadrático & $\mathrm{C}+\mathrm{L}$ & $0.907^{*}$ & 0.822 & 4.188 & 5.814 & 0.946 & 0.857 \\
11 & Cúbico & $\mathrm{C}+\mathrm{L}$ & $0.903^{*}$ & 0.815 & 4.251 & 5.936 & 0.945 & 0.853 \\
12 & Potencial & $\mathrm{C}+\mathrm{L}$ & $0.907^{*}$ & 0.822 & 4.280 & 5.848 & 0.943 & 0.855 \\
13 & Exponencial & $\mathrm{C}+\mathrm{L}$ & $0.900^{*}$ & 0.810 & 4.387 & 6.123 & 0.945 & 0.851 \\
\hline
\end{tabular}

"Significativo a $1 \%$ de probabilidade de erro pelo teste $\mathrm{t}$

Os modelos linear, linear sem intercepto, quadrático e potencial com base na relação $(\mathrm{CxL})$, para área reais de 25, 50 e $75 \mathrm{~cm}^{2}$, esses modelos estimam área foliares de (26,76; 48,55 e 70,34 cm²), $(27,72 ; 48,40$ e $\left.69,07 \mathrm{~cm}^{2}\right),\left(26,05 ; 48,82\right.$ e $\left.71,60 \mathrm{~cm}^{2}\right)$ e $\left(26,83 ; 48,32\right.$ e $\left.69,81 \mathrm{~cm}^{2}\right)$, respectivamente (Figura 1 e Figura 2).

Figura 1. Modelo de regressão linear (A) e linear sem intercepto (C) e seus respectivos testes (B) e (D), obtidos entre o produto $(\mathrm{CxL})$ da folha e a área do limbo foliar de limoeiro. Capitão Poço - PA, 2012.
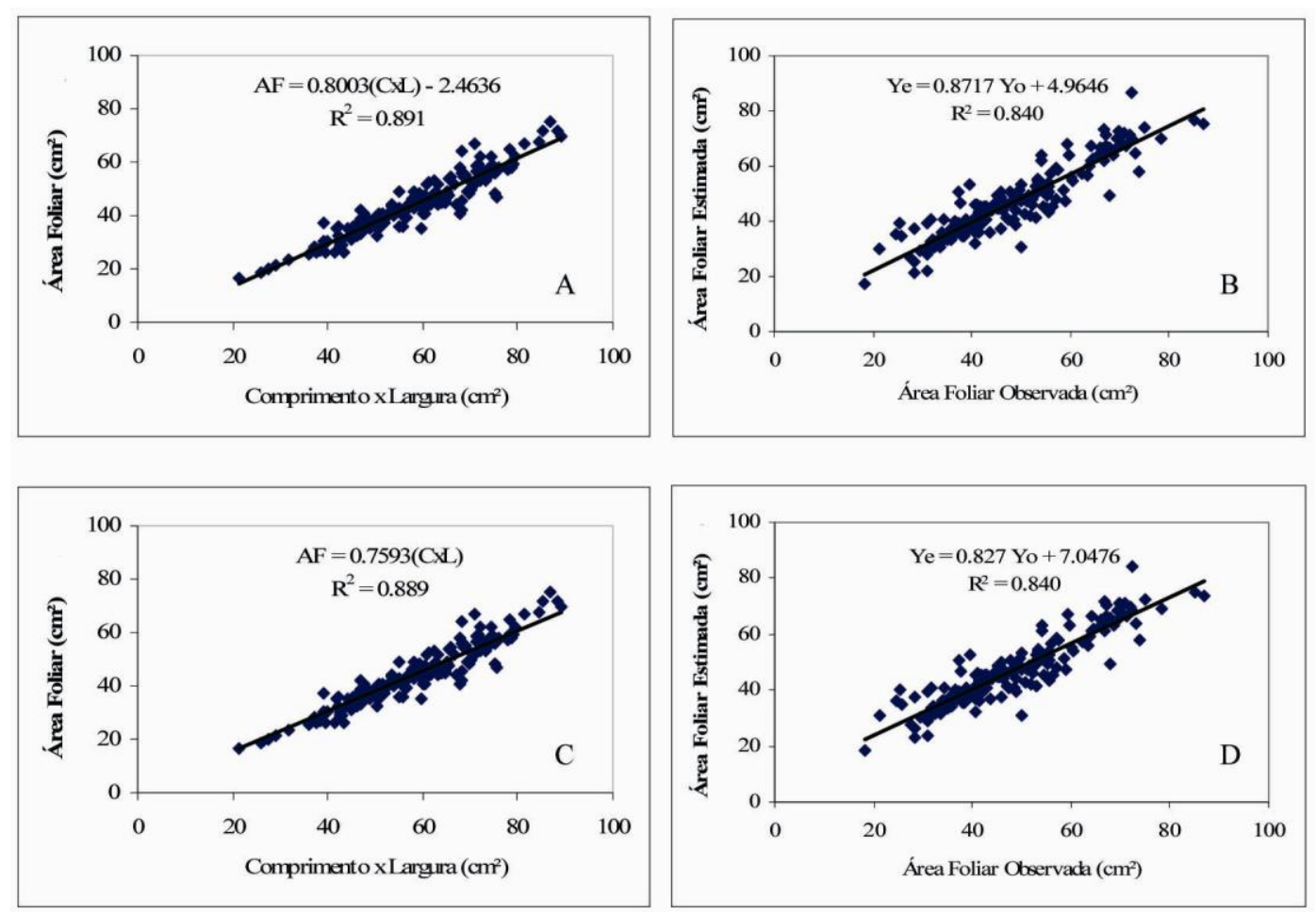
Figura 2. Modelo de regressão quadrático $(E)$ e potência $(G)$ e seus respectivos testes $(F)$ e $(G)$, obtidos entre o produto $(\mathrm{CxL})$ da folha e a área do limbo foliar de limoeiro. Capitão Poço - PA, 2012.
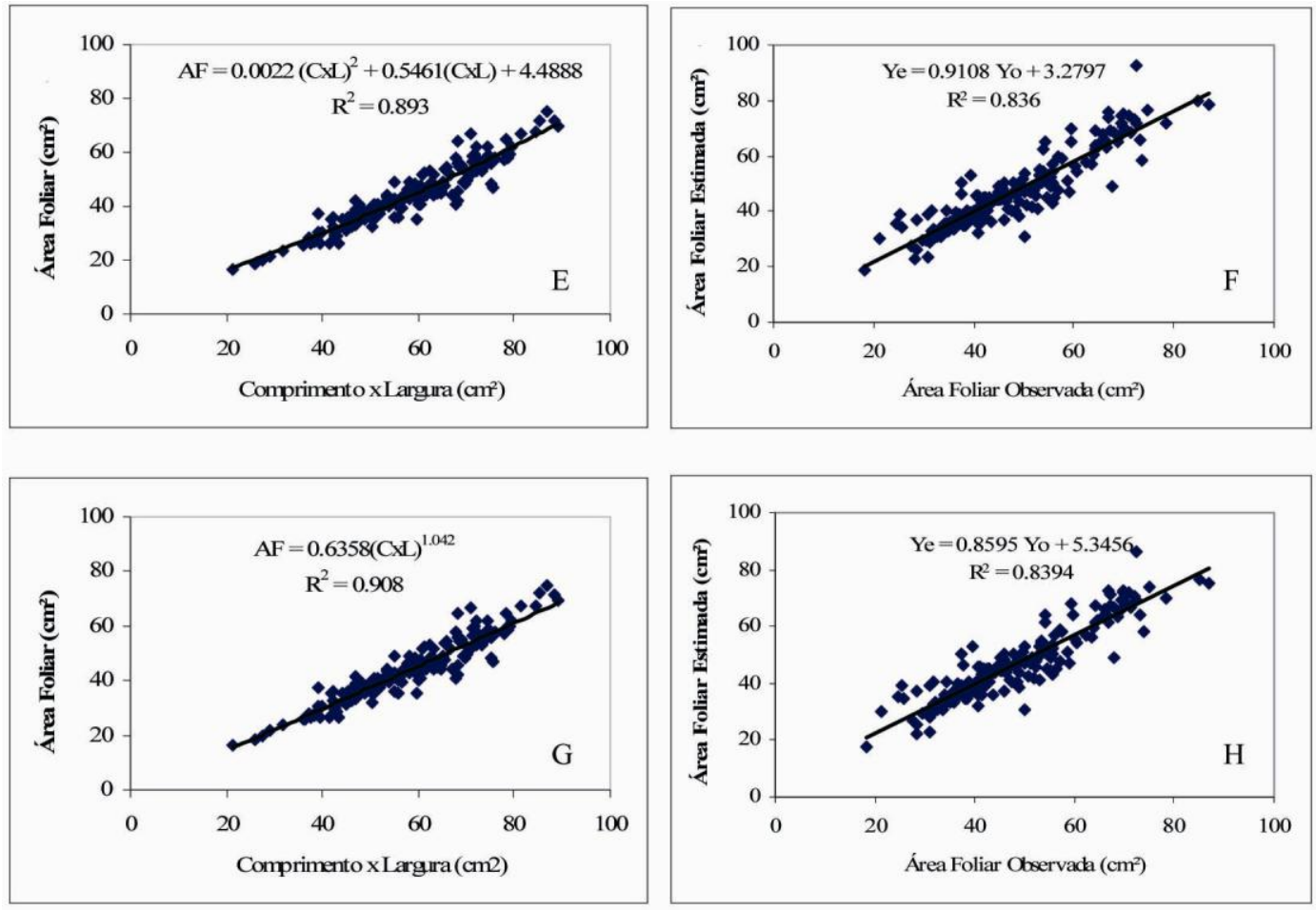

Para Queiroga et al. (2003), a precisão de equações para estimativa de área foliar, dentre as várias possibilidades ou de combinações entre parâmetros dimensionais e modelos de regressão, relaciona-se não só com o formato da folha, mas também com a sua variação durante o crescimento da planta.

Resultados semelhantes aos encontrados nesse trabalho foram verificados por: Figueiredo et al. (2012) em que a área foliar do feijoeiro comum pode ser estimada a partir do modelo linear com boa precisão. Silva et al. (2002), verificaram que as estimativas da área foliar de plantas de gergelim são mais precisas quando se usam ambas as dimensões do limbo foliar.

Segundo Toebe et al. (2010), verificaram maior precisão na estimativa de área foliar de Crambe através do modelo tipo potência $\left(R^{2} \geq 0,9805\right)$, seguido do modelo quadrático $\left(R^{2} \geq 0,9702\right)$.

Pinto et al. (2007) na cultura da maniçoba (Ageratum conyzoides L.), mostraram que o modelo matemático linear sem intercepto, utilizando o produto entre o comprimento e a largura da folha foi o mais preciso.

Para o capim-aruana (GALZERANO et al., 2012), obteve a melhor estimativa de área foliar quando se utilizou do produto do comprimento pela máxima largura do limbo foliar.

\section{CONCLUSÃO}

Medidas de área foliar do limoeiro 'Cravo' podem ser estimadas a partir de equações linear, linear sem intercepto, quadrática e potencial considerando a relação (comprimento x largura) como variável independente.

A área foliar pode ser estimada pelas seguintes equações: $\mathrm{AF}=0.8003(\mathrm{CxL})-2.4636, \mathrm{AF}=$ $0.7593(\mathrm{CxL}), \mathrm{AF}=0.0022(\mathrm{CxL})^{2}+0.5461(\mathrm{CxL})+4.4888 \mathrm{e} \mathrm{AF}=0.6358(\mathrm{CxL})^{1.042}$. 


\section{REFERÊNCIAS}

BIANCO, S. et al. Estimativa da área foliar de Cissampelos glaberrima L. usando dimensões lineares do limbo foliar. Planta Daninha, Viçosa, v.20, n.3, p.353-356, 2002.

BIANCO, S. et al. Estimativa da área foliar de Ipomoea hederifolia e Ipomoea nil Roth. Usando dimensões lineares do limbo foliar. Planta Daninha, Viçosa, MG, v. 25, n.2, p.325-329, 2007.

BIANCO, S. et al. Estimativa da área foliar de Tridax procumbens usando dimensões lineares do limbo foliar. Planta Daninha, Viçosa, v.22, n.2, p.247-250, 2004.

BIANCO, S. et al. Estimativa da área foliar de Typha latifolia L. usando dimensões lineares do limbo foliar. Planta Daninha, Viçosa, v.21, n.2, p.257-261, 2003.

BLANCO, F. F.; FOLEGATTI, M. V.; Estimation of leaf area for greenhouse cucumber by linear measurements under salinity and grafting. Scientia Agricola, Piracicaba, v. 62, n. 4, p.305-309, 2005.

CAMARGO, A. P.; SENTELHAS, P. C. Avaliação do desempenho de diferentes métodos de estimativa da evapotranspiração potencial no estado de São Paulo, Brasil. Revista Brasileira de Agrometeorologia, Santa Maria, v.5, n.1, p.89-97, 1997.

FIGUEIREDO, E. S.; SANTOS, M. E.; GARCIA, A. Modelos de determinação não destrutivo da área foliar do feijoeiro comum (Phaseolus vulgaris L.). Nucleus, Ituverava, v.9, n.1, p.79-84, 2012.

GALZERANO, L. et al. Medidas lineares na estimativa da área foliar do capim-aruana. Nucleus Animalium, Ituverava, v.4, n.1, p.79-82, 2012.

LARCHER, W. Ecofisiologia vegetal. São Carlos: Rima, 2000. 531 p.

LIMA, C. J. G. S. et al. Modelos matemáticos para estimativa de área foliar de feijão caupí. Revista Caatinga, Mossoró, v.21, n.1, p.120-127, 2008.

LUCENA, R. R. M. et al. Medição de área foliar de aceroleira. Revista Caatinga, Mossoró, v.24, n.2, p 40-45, 2011.

MARROCOS, S. T. P. et al. Análise comparativa de métodos de estimativa de área foliar em beterraba.

Revista Verde, Mossoró, v.5, n.5, p.140-146, 2010.

MONTEIRO, J. E. B. A. et al. Estimação da área foliar do algodoeiro por meio de dimensões e massa das folhas. Bragantia, Campinas, v.64, n.1, p.15-24, 2005.

PEREIRA, A.R.; VILA NOVA, N.A.; SEDIYAMA, R. Evapotranspiração. Piracicaba:

FEALQ/ESALQ/USP, 1997.70p.

PINTO, M. S. C. et al. Modelo para estimativa da área foliar da maniçoba. Revista Ciência Agronômica, Fortaleza, v.38, n.4, p.391-395, 2007.

QUEIROGA, J. L. et al. Estimativa da área foliar do feijão-vagem (Phaseolus vulgaris L.) por meio da largura máxima do folíolo central. Horticultura Brasileira, Brasília, v.21, n.1, p.64-68, 2003.

SCHWART, G. Manejo sustentável de florestas secundárias: espécies potenciais no Nordeste do Pará, Brasil. Amazônia: Ciência ; Desenvolvimento, Belém, v.3, n.5, p.125-147, 2007.

SEVERINO, L. S. et al. Método para determinação da área foliar da momoneira. Revista Brasileira de Oleaginosas e Fibrosas, Campina Grande, v.8, n.1, p.753-762, 2004.

SILVA, L. C. et al. Um método simples para se estimar área foliar de plantas de gergelim (Sesamum indicum L.). Revista Brasileira de Oleaginosas e Fibrosas, Campina Grande, v.6, n.1, p.491-496, 2002. 
TAIZ, L.; ZEIGER, E. Fisiologia vegetal. 3. ed. Porto Alegre: Artmed, 2004. 719p.

TOEBE, M. et al. Estimativa da área foliar de Crambe abyssinica por discos foliares e por fotos digitais. Ciência Rural, Santa Maria, v. 40, n.2, p 445-448, 2010.

TONET, F. P. et al.Variedades de limão comercializadas em Cuiabá - MT, origem, preferência, demanda e perdas. UNICiências, v.14, n.2, p.31-46, 2010.

WILLMOTT, C. J. On the validation of models. Physical Geography, v.2, n.2, p.184-194, 1981. 